\title{
ADAPTIVE WAVELET METHODS FOR SADDLE POINT PROBLEMS*
}

\author{
Stephan Dahlke $^{1}$, Reinhard Hochmuth $^{2}$ and Karsten Urban ${ }^{1}$
}

\begin{abstract}
Recently, adaptive wavelet strategies for symmetric, positive definite operators have been introduced that were proven to converge. This paper is devoted to the generalization to saddle point problems which are also symmetric, but indefinite. Firstly, we investigate a posteriori error estimates and generalize the known adaptive wavelet strategy to saddle point problems. The convergence of this strategy for elliptic operators essentially relies on the positive definite character of the operator. As an alternative, we introduce an adaptive variant of Uzawa's algorithm and prove its convergence. Secondly, we derive explicit criteria for adaptively refined wavelet spaces in order to fulfill the LadyshenskajaBabuška-Brezzi (LBB) condition and to be fully equilibrated.
\end{abstract}

Mathematics Subject Classification. 65J10, 65T60, 42C40.

Received: April 16, 1999. Revised: March 31, 2000.

\section{INTRODUCTION}

The variational formulation of many problems in mechanics, physics and technology leads to a saddle point problem. For example, mixed methods are widely used in structural and fluid mechanics, [5, 7]. Although significant progress has been made in the numerical treatment of such equations, they still form a class of challenging problems. The indefinite character of saddle point problems requires some care in the choice of the discretization in order to obtain a stable numerical method. Moreover, the efficient solvers that are available for symmetric positive definite operators, have to be appropriately modified.

In addition, many saddle point problems show a large scale behaviour in the sense that the solution has some global (low frequency) part and well localized (high frequency) details which may come from singularities of the problem data such as jumping coefficients, non-smooth domains and right-hand sides. These problems demand the use of adaptive strategies in order to resolve the local details of the solution up to a given accuracy while preserving efficiency.

Keywords and phrases. Adaptive schemes, a posteriori error estimates, multiscale methods, wavelets, saddle point problems, Uzawa's algorithm.

* The work of the first two authors has been supported by Deutsche Forschungsgemeinschaft (DFG) under Grants Da 117/13-1 and Ho 1846/1-1, respectively. Moreover, this work was supported by the European Commission within the TMR project (Training and Mobility for Researchers) Wavelets and Multiscale Methods in Numerical Analysis and Simulation, No. ERB FMRX CT98 018T4 and by the German Academic Exchange Service (DAAD) within the VIGONI-Project MultilevelZerlegungsverfahren für Partielle Differentialgleichungen. This paper was partially written when the third author was in residence at the Istituto di Analisi Numerica del C.N.R. in Pavia, Italy.

1 RWTH Aachen, Institut für Geometrie und Praktische Mathematik, Templergraben 55, 52056 Aachen, Germany. e-mail: \{dahlke, urban\}@igpm.rwth-aachen.de

2 FU Berlin, FB Mathematik, Arnimallee 2-6, 14195 Berlin, Germany.

e-mail: hochmuth@math.fu-berlin.de 
In this paper, we treat these problems by means of wavelet analysis. The first applications of wavelet methods were in image and signal processing. During the last years, they have also been shown to offer some potential for the numerical treatment of partial differential and integral equations, see $[8,14,15]$ and the references therein. Among them, the maybe most important features for adaptive solution methods for saddle point problems are:

- Convergent and efficient adaptive wavelet methods for positive definite problems.

- Construction of adapted wavelet bases.

Let us describe this in more detail. Recently, an adaptive wavelet strategy has been introduced for symmetric positive definite operators, [10]. It was proven there that this strategy gives rise to a convergent adaptive algorithm. The original method in [10] was somewhat modified in [9] resulting in a strategy that in addition was proven to be asymptotically optimal efficient.

The construction of (biorthogonal) wavelet bases leaves some freedom that can be exploited to fulfill additional requirements that, e.g., are forced by the problem to solve. As an example, we mention the construction of divergence- and curl-free wavelets [20,22,23] and of wavelet trial spaces for the Stokes problem that fulfill the Ladyshenskaja-Babuška-Brezzi (LBB) condition, [16].

From what is said above it seems natural for us to consider the construction of adaptive wavelet strategies for saddle point problems. In this paper, we focus on two main questions:

- Is there an adaptive wavelet strategy for saddle point problems that can be proven to converge?

- Is it possible to derive general and explicit criteria for adaptive wavelet discretizations of saddle point problems in order to fulfill (LBB)? Moreover, is the same possible for the so called full equilibrium property (FEP) (see Def. 2.3 below)?

We answer both questions positively in this paper. After collecting some preliminaries in Section 2, we introduce an a posteriori error analysis in Section 3 which is in fact a generalization of the result in [10]. Also, the adaptive refinement strategy in [10] can be generalized to saddle point problems. It is still an open problem to prove the convergence of this particular strategy for saddle point problems. Alternatively, we propose an adaptive variant of Uzawa's algorithm in Section 4 and we prove its convergence. Also this method may be viewed as a generalization of the results in [10] since the adaptive Uzawa algorithm uses a convergent adaptive strategy for the elliptic part as a main ingredient.

In order to answer the second question from above, we prove general and explicit criteria for (LBB) and (FEP) in Section 5 in the context of adaptively chosen biorthogonal wavelet bases. These criteria have been detailed for two concrete examples, namely:

- the Stokes problem, and

- second order elliptic boundary value problems where boundary conditions are appended by Lagrange multipliers,

in the preprint version of this paper [11].

\section{Preliminaries}

In this section, we collect all the auxiliary facts on both, saddle point problems and wavelets, that will be needed in the sequel.

\subsection{Setting}

We consider the following saddle point problem: We are given two Hilbert spaces $X$ and $M$, two continuous bilinear forms

$$
a: X \times X \rightarrow \mathbb{R}, \quad b: X \times M \rightarrow \mathbb{R}
$$


and $f \in X^{\prime}$ as well as $g \in M^{\prime}$. Here, $Y^{\prime}$ denotes the dual space of a Banach space $Y$. Moreover, we assume $X \subseteq H_{X}, M \subseteq H_{M}$, where $H_{X}, H_{M}$ are Hilbert spaces such that

$$
X \hookrightarrow H_{X} \hookrightarrow X^{\prime}, \quad M \hookrightarrow H_{M} \hookrightarrow M^{\prime}
$$

Then, one has to determine a pair $[u, p] \in X \times M$ such that

$$
\begin{array}{lll}
a(u, v)+b(v, p) & =\langle f, v\rangle_{X^{\prime} \times X} & \text { for all } v \in X \\
b(u, q) & =\langle g, q\rangle_{M^{\prime} \times M} & \text { for all } q \in M
\end{array}
$$

where $\langle\cdot, \cdot\rangle_{Y^{\prime} \times Y}$ denotes the dual pairing. In general, we assume the bilinear form $a(\cdot, \cdot)$ to be elliptic on the subspace

$$
V:=\{v \in X: b(v, q)=0 \text { for all } q \in M\} \subset X
$$

i.e., there exists a constant $\alpha>0$ such that

$$
a(v, v) \geq \alpha\|v\|_{X}^{2}
$$

holds for all $v \in V$. For the analysis of our adaptive Uzawa algorithm however, we will need ellipticity on all of $X$. Since we are ultimately interested in problems of the kind (2.2) that are uniquely solvable, we finally assume that $X$ and $M$ fulfill the inf-sup condition:

$$
\inf _{q \in M} \sup _{v \in X} \frac{b(v, q)}{\|v\|_{X}\|q\|_{M}} \geq \beta
$$

for some constant $\beta>0$.

The following equivalent formulation will be very useful for our analysis. Defining the operators

$$
\begin{array}{lllll}
A: X \rightarrow X^{\prime}, & \langle A u, v\rangle_{X^{\prime} \times X}:= & a(u, v), & v \in X, \\
B: X \rightarrow M^{\prime}, & \langle B u, q\rangle_{M^{\prime} \times M}:= & b(u, q), & q \in M, \\
B^{\prime}: M \rightarrow X^{\prime}, & \left\langle B^{\prime} p, v\right\rangle_{X^{\prime} \times X}:= & b(v, p), & v \in X,
\end{array}
$$

the Problem (2.2) is equivalent to find $[u, p] \in X \times M=: \mathcal{H}$ such that

$$
\begin{array}{lll}
A u+B^{\prime} p & =f \quad \text { in } X^{\prime}, \\
B u & =g \quad \text { in } M^{\prime} .
\end{array}
$$

If (2.2) is well-posed, the operator

$$
\mathcal{A}:=\left(\begin{array}{cc}
A & B^{\prime} \\
B & 0
\end{array}\right)
$$

is boundedly invertible with respect to the usual graph norm, i.e., there exist constants $c_{\mathcal{A}}, C_{\mathcal{A}}$ such that

$$
c_{\mathcal{A}}\|\mathcal{A}([u, p])\|_{\mathcal{H}^{\prime}} \leq\|[u, p]\|_{\mathcal{H}} \leq C_{\mathcal{A}}\|\mathcal{A}([u, p])\|_{\mathcal{H}^{\prime}}
$$

where $\|[u, p]\|_{\mathcal{H}}^{2}:=\|u\|_{X}^{2}+\|p\|_{M}^{2}$. We will often use the notation $a \lesssim b$ to abbreviate $a \leq c b$ with some constant $c>0$, and for $a \lesssim b \lesssim a$, we write $a \sim b$. Hence, (2.7) may also be expressed by $\|\mathcal{A}([u, p])\|_{\mathcal{H}^{\prime}} \sim\|[u, p]\|_{\mathcal{H}}$. 
The Schur complement. In many cases of interest, the operator $A$ is invertible and it makes sense to consider the operator $S:=B A^{-1} B^{\prime}$, which is known as the Schur complement. Then, we define the energy norm on $X \times M$ for the operator $\mathcal{A}$ by

$$
\|[v, q]\|_{\mathcal{A}}^{2}:=\|v\|_{A}^{2}+\|q\|_{S}^{2}, \quad[v, q] \in X \times M
$$

where $\|\cdot\|_{A},\|\cdot\|_{S}$ denote the energy norms corresponding to A and the Schur complement $S$, respectively. We include the proof of the following fact for completeness and convenience.

Proposition 2.1. If $A$ is elliptic on all of $X, A$ and $B^{\prime}$ are bounded and the inf-sup condition (2.4) holds, then

$$
\|q\|_{S} \sim\|q\|_{M}, \quad q \in M
$$

Proof. We first establish the upper estimate. Due to the boundedness of $B^{\prime}$ and of $A^{-1}$ (which is a consequence of the boundedness and ellipticity of $A$ ), we have

$$
\begin{aligned}
\|q\|_{S}^{2} & =\langle S q, q\rangle_{M^{\prime} \times M}=\left\langle A^{-1} B^{\prime} q, B^{\prime} q\right\rangle_{X \times X^{\prime}} \leq\left\|A^{-1} B^{\prime} q\right\|_{X}\left\|B^{\prime} q\right\|_{X^{\prime}} \\
& \lesssim\left\|B^{\prime} q\right\|_{X^{\prime}}^{2} \lesssim\|q\|_{M}^{2} .
\end{aligned}
$$

To show the lower estimate, we use the boundedness and the ellipticity of $A$ as well as the inf-sup condition:

$$
\|q\|_{S}^{2}=\left\langle A^{-1} B^{\prime} q, B^{\prime} q\right\rangle_{X \times X^{\prime}} \gtrsim\left\|B^{\prime} q\right\|_{X^{\prime}}^{2} \gtrsim\|q\|_{M}^{2}
$$

This completes the proof.

This result shows the equivalence of $\|\cdot\|_{\mathcal{A}}$ to the graph norm $\|\cdot\|_{\mathcal{H}}$. Since we always assume that (2.2) is well-posed, we also have that $\mathcal{A}$ is boundedly invertible with respect to $\|\cdot\|_{\mathcal{A}}$, i.e.,

$$
\|\mathcal{A}([v, q])\|_{X^{\prime} \times M^{\prime}} \sim\|[v, q]\|_{X \times M} \sim\|[v, q]\|_{\mathcal{A}}
$$

which is a consequence of Proposition 2.1 and the well-posedness of (2.2).

An equivalent formulation. An equivalent formulation of (2.2) can be introduced by the bilinear form

$$
\mathcal{L}([u, p],[v, q]):=a(u, v)+b(v, p)+b(u, q),
$$

which is defined for $[u, p],[v, q] \in X \times M$. Now, $(2.2)$ can be rewritten in terms of the bilinear form $\mathcal{L}(\cdot, \cdot)$ : Given $f \in X, g \in M^{\prime}$, find a pair $[u, p] \in X \times M$ such that

$$
\mathcal{L}([u, p],[v, q])=\langle f, v\rangle_{X^{\prime} \times X}+\langle g, q\rangle_{M^{\prime} \times M}, \quad[v, q] \in X \times M
$$

A short reflection shows that (2.2) and (2.11) are indeed equivalent: The conclusion from (2.2) to (2.11) can be made by adding up the two equations in (2.2). For the other direction, one can take test functions with $q=0$ and $v=0$, respectively, to obtain (2.2). The following lemma shows that $\mathcal{L}$ also fulfills an inf-sup condition. We will use this fact later on.

Lemma 2.2. ([19]) There exists a constant $\tilde{\beta} \in \mathbb{R}^{+}$with

$$
\inf _{[u, p] \in X \times M} \sup _{[v, q] \in X \times M} \frac{\mathcal{L}([u, p],[v, q])}{\left(\|u\|_{X}+\|p\|_{M}\right)\left(\|v\|_{X}+\|q\|_{M}\right)} \geq \tilde{\beta}>0 .
$$




\subsection{Multiscale methods and wavelets}

Let us now summarize the basic notation for multiscale methods that are needed in this paper. For a survey of multiscale methods and wavelets, we refer to $[8,14]$.

Given some Hilbert space $H$ and a sequence $\Delta_{j}$ of (finite) sets of indices, we call a system of functions $\Phi_{j}:=\left\{\varphi_{j, k}: k \in \Delta_{j}\right\}, j \geq j_{0}$, (primal) single scale system, if $\Phi_{j}$ is refinable, i.e. there exists a matrix $M_{j, 0} \in \mathbb{R}^{\left|\Delta_{j+1}\right| \times\left|\Delta_{j}\right|}$ such that

$$
\Phi_{j}=M_{j, 0}^{T} \Phi_{j+1}
$$

Here, $j_{0} \in \mathbb{N}$ denotes some coarse level. Equation (2.13) in particular implies that the induced spaces

$$
S_{j}:=S\left(\Phi_{j}\right):=\operatorname{span}\left(\Phi_{j}\right)
$$

are nested: $S_{j} \subset S_{j+1}$. We always assume that the union of all $S_{j}$ is dense in $H$. Moreover, we assume the existence of a dual single scale system $\tilde{\Phi}_{j}=\left\{\tilde{\varphi}_{j, k} ; k \in \Delta_{j}\right\}$, such that

$$
\left\langle\Phi_{j}, \tilde{\Phi}_{j}\right\rangle:=\left(\left(\varphi_{j, k}, \tilde{\varphi}_{j, k^{\prime}}\right)_{H}\right)_{k, k^{\prime} \in \Delta_{j}}=I d
$$

where $I d$ denotes the identity matrix of corresponding size.

Biorthogonal wavelet spaces $W_{j}, \tilde{W}_{j}$ are then defined by

$$
W_{j}:=S_{j+1} \ominus S_{j}, \tilde{W}_{j}:=\tilde{S}_{j+1} \ominus \tilde{S}_{j}, S_{j} \perp \tilde{W}_{j}, \tilde{S}_{j} \perp W_{j}
$$

where the orthogonality is to be understood with respect to the $H$-inner product. Constructing biorthogonal wavelets then amounts to finding bases

$$
\Psi_{j}:=\left\{\psi_{j, k}: k \in \nabla_{j}\right\}, \tilde{\Psi}_{j}:=\left\{\tilde{\psi}_{j, k}: k \in \nabla_{j}\right\}, \quad\left(\nabla_{j}:=\Delta_{j+1} \backslash \Delta_{j}\right)
$$

of $W_{j}, \tilde{W}_{j}$, respectively, such that

$$
\left\langle\Psi_{j}, \tilde{\Psi}_{j}\right\rangle=I d
$$

and the collections

$$
\Psi:=\left\{\psi_{\lambda}: \lambda \in \nabla\right\}, \quad \tilde{\Psi}:=\left\{\tilde{\psi}_{\lambda}: \lambda \in \nabla\right\}, \quad \nabla:=\left\{\lambda=(j, k): j \geq j_{0}-1, k \in \nabla_{j}\right\}
$$

(with $\Psi_{j_{0}-1}:=\Phi_{j_{0}}, \tilde{\Psi}_{j_{0}-1}:=\tilde{\Phi}_{j_{0}}$ ) form Riesz bases for $H$, i.e., they form a basis for $H$ and the following norm equivalence holds

$$
\left\|\boldsymbol{d}^{T} \Psi\right\|_{H}=\left\|\sum_{\lambda \in \nabla} d_{\lambda} \psi_{\lambda}\right\|_{H} \sim\|\boldsymbol{d}\|_{\ell_{2}(\nabla)}=\left(\sum_{\lambda \in \nabla}\left|d_{\lambda}\right|^{2}\right)^{1 / 2}
$$

We will frequently use the abbreviation

$$
|\lambda|:=j \text { for } \lambda=(j, k)
$$

to indicate the level of a wavelet function. Often, $\Psi, \tilde{\Psi}$ will be termed biorthogonal wavelet system or simply multiscale basis. In many cases, an equation similar to (2.19) also holds for a whole range of Sobolev or Besov 
spaces including $H$ (see [13] and also (2.23), (2.24) below). In the sequel, we shall always assume that all functions $\psi_{\lambda}$ are compactly supported and that

$$
\operatorname{diam}\left(\square_{\lambda}\right) \sim 2^{-|\lambda|}, \quad \square_{\lambda}:=\operatorname{supp} \psi_{\lambda} .
$$

For any subset $\Lambda \subset \nabla$, we define the corresponding set of wavelets by

$$
\Psi_{\Lambda}:=\left\{\psi_{\lambda}: \lambda \in \Lambda\right\}, \quad \tilde{\Psi}_{\Lambda}:=\left\{\tilde{\psi}_{\lambda}: \lambda \in \Lambda\right\},
$$

and the induced spaces by $S_{\Lambda}:=S\left(\Psi_{\Lambda}\right)$ and $\tilde{S}_{\Lambda}:=S\left(\tilde{\Psi}_{\Lambda}\right)$.

\subsection{Multiscale discretization of saddle point problems}

In order to discretize (2.2), we want to use trial and test spaces that are induced by multiscale bases. To be more specific, we assume that there exist wavelet bases $\Psi=\left\{\psi_{\lambda}: \lambda \in \nabla^{X}\right\}$ and $\Theta=\left\{\vartheta_{\mu}: \mu \in \nabla^{M}\right\}$ that form Riesz bases for $H_{X}$ and $H_{M}$, respectively, see (2.1). In the sequel, we shall restrict ourselves mainly to the case that $X$ and $M$ are Hilbertian Sobolev spaces defined on suitable domains or manifolds $\Omega_{1} \subset \mathbb{R}^{n}, \Omega_{2} \subset \mathbb{R}^{m}$, i.e.,

$$
X=H^{t}\left(\Omega_{1}\right), \quad M=H^{s}\left(\Omega_{2}\right), \quad s, t \in \mathbb{R} .
$$

Then, we also assume that the Riesz bases give rise to the following norm equivalences (recall (2.20)):

$$
\begin{aligned}
\left\|\boldsymbol{d}^{T} \Psi\right\|_{\tau, \Omega_{1}}^{2} & \sim \sum_{\lambda \in \nabla^{X}} 2^{2 \tau|\lambda|} d_{\lambda}^{2}, & \tau \in[-t, t], \\
\left\|\boldsymbol{c}^{T} \Theta\right\|_{\varsigma, \Omega_{2}}^{2} & \sim \sum_{\mu \in \nabla^{M}} 2^{2 \varsigma|\mu|} c_{\mu}^{2}, & \varsigma \in[-s, s],
\end{aligned}
$$

where $\|\cdot\|_{m, \Omega}$ denotes the norm in the Sobolev space $H^{m}(\Omega), m \in \mathbb{R}$. Since it should be clear from the context, we will omit the dependencies of the norms on $\Omega_{1}$ and $\Omega_{2}$, respectively, in the sequel. Now, the trial spaces $\left(X_{\Lambda}, M_{\Lambda}\right) \subset(X, M)$ are defined by a pair of index sets

$$
\Lambda:=\left(\Lambda^{X}, \Lambda^{M}\right) \subset\left(\nabla^{X}, \nabla^{M}\right) .
$$

The LBB condition. It is well-known that trial spaces for the stable numerical solution of (2.2) need to fulfill the Ladyshenskaja-Babuška-Brezzi (LBB) condition

$$
\inf _{q_{\lambda} \in M_{\Lambda}} \sup _{v_{\lambda} \in X_{\Lambda}} \frac{b\left(v_{\lambda}, q_{\lambda}\right)}{\left\|v_{\lambda}\right\|_{X}\left\|q_{\lambda}\right\|_{M}} \geq \beta
$$

for some constant $\beta>0$ independent of $\Lambda$.

Full equilibrium property. For the numerical treatment of saddle point problems as well as for the analysis of discretizations, the following property is very useful.

Definition 2.3. A discretization $\left(X_{\Lambda}, M_{\Lambda}\right)$ is said to have the full equilibrium property (FEP) if for $u_{\Lambda} \in X_{\Lambda}$ the equality $b\left(u_{\Lambda}, q_{\Lambda}\right)=0$ for all $q_{\Lambda} \in M_{\Lambda}$ already implies that $u_{\Lambda} \in V$, i.e., $b\left(u_{\Lambda}, q\right)=0$ for all $q \in M$. The spaces are also called equilibrated.

Roughly speaking, this means that $\operatorname{Ker} B_{\Lambda} \subset \operatorname{Ker} B$, which is, of course, a very strong property. There are many different names in the literature for this property. We choose (FEP), which is used in applications of mixed methods in structural mechanics (see, e.g., [7]). 


\section{A POSTERIORI ERROR ESTIMATES AND A REFINEMENT STRATEGY}

As already mentioned, a convergent adaptive wavelet strategy for symmetric, positive definite operators has been introduced in [10]. However, a closer look at the proofs in [10] shows that the results concerning the construction of an adaptive refinement strategy (without proof of convergence) can easily be generalized to a more general setting (including saddle point problems), see also [3]. In this section, we will therefore briefly review the relevant results but we omit the proofs since they can easily be deduced from [10]. Finally, we describe the application of these results to saddle point problems.

\subsection{The general setting}

Let us now describe the setting that we consider in this section. Let $L: H \rightarrow H^{\prime}$ be a linear boundedly invertible operator, i.e.,

$$
c_{L}\|L x\|_{H^{\prime}} \leq\|x\|_{H} \leq C_{L}\|L x\|_{H^{\prime}}, \quad x \in H
$$

where $0<c_{L} \leq C_{L}$ are absolute constants and $H$ is some Hilbert space.

We consider the problem

$$
L x=z
$$

for a given $z \in H^{\prime}$. Moreover, we assume the existence of biorthogonal wavelet bases $\Psi=\left\{\psi_{\lambda}: \lambda \in \nabla\right\}$, $\tilde{\Psi}=\left\{\tilde{\psi}_{\lambda}: \lambda \in \nabla\right\}$, such that $\tilde{\Psi}$ characterizes $H^{\prime}$, i.e., there exist absolute constants $0<c_{\Psi} \leq C_{\Psi}$, such that

$$
c_{\Psi}\left(\sum_{\lambda \in \nabla}\left|\gamma_{\lambda}\left\langle y, \psi_{\lambda}\right\rangle\right|^{2}\right)^{1 / 2} \leq\|y\|_{H^{\prime}} \leq C_{\Psi}\left(\sum_{\lambda \in \nabla}\left|\gamma_{\lambda}\left\langle y, \psi_{\lambda}\right\rangle\right|^{2}\right)^{1 / 2},
$$

for any $y \in H^{\prime}$, where $\gamma_{\lambda}$ are suitable weight factors and $\langle\cdot, \cdot\rangle$ denotes the dual pairing of $H$ and $H^{\prime}$.

Now, we consider the Galerkin approximation of (3.2), i.e., we look for some $x_{\Lambda} \in S_{\Lambda}:=S\left(\Psi_{\Lambda}\right)$ such that

$$
L_{\Lambda} x_{\Lambda}=z_{\Lambda}
$$

where $L_{\Lambda}, z_{\Lambda}$ are the usual Galerkin projections of $L$ and $z$, i.e., $L_{\Lambda}=\left(\left\langle L \psi_{\lambda^{\prime}}, \psi_{\lambda}\right\rangle\right)_{\lambda, \lambda^{\prime} \in \Lambda}$ and $z_{\Lambda}=\left(\left\langle z, \psi_{\lambda}\right\rangle\right)_{\lambda \in \Lambda}$, respectively. We will always assume that the Problem (3.4) is well-posed which, e.g., is fulfilled if

$$
\inf _{v_{\Lambda} \in S_{\Lambda}} \sup _{w_{\Lambda} \in S_{\Lambda}} \frac{\left\langle L v_{\Lambda}, w_{\Lambda}\right\rangle}{\left\|v_{\Lambda}\right\| H\left\|w_{\Lambda}\right\|_{H}} \geq \gamma>0
$$

see, e.g., [5]. Note that $x_{\Lambda}$ is indeed the Galerkin solution, i.e., we have the Galerkin orthogonality

$$
\left\langle L\left(x-x_{\Lambda}\right), y_{\Lambda}\right\rangle=0 \quad \text { for all } \quad y_{\Lambda} \in S_{\Lambda} .
$$

\subsection{A posteriori error estimates}

Now, using (3.1), (3.3) and the Galerkin orthogonality already gives rise to an a posteriori error estimate:

Proposition 3.1. Under the above assumptions, one has for

$$
\delta_{\lambda}:=\left|\gamma_{\lambda}\left\langle L\left(x-x_{\Lambda}\right), \psi_{\lambda}\right\rangle\right|, \quad \lambda \in \nabla,
$$

the estimate

$$
c_{L} c_{\Psi}\left(\sum_{\lambda \in \nabla \backslash \Lambda} \delta_{\lambda}^{2}\right)^{1 / 2} \leq\left\|x-x_{\Lambda}\right\|_{H} \leq C_{L} C_{\Psi}\left(\sum_{\lambda \in \nabla \backslash \Lambda} \delta_{\lambda}^{2}\right)^{1 / 2} .
$$


Equation (3.7) states that we already have an efficient and reliable error estimator. However, this is numerically useless, since $\nabla \backslash \Lambda$ is a set of infinite cardinality so that the estimator is not accessible. Hence, the idea is to reduce the infinite sums in (3.7) to finite ones allowing some additional error that is under control. In order to do so, we have to pose one more assumption on $L$ and $\Psi$. To be precise, we call $L$ quasi sparse w.r.t. $\Psi$, if

$$
2^{-\left(\left|\lambda^{\prime}\right|+|\lambda|\right) t}\left|\left\langle L \psi_{\lambda^{\prime}}, \psi_{\lambda}\right\rangle\right| \lesssim \frac{2^{-\varsigma|| \lambda|-| \lambda^{\prime}||}}{\left(1+2^{\min \left(|\lambda|,\left|\lambda^{\prime}\right|\right)}\right) \operatorname{dist}\left(\square_{\lambda}, \square_{\lambda^{\prime}}\right)^{\tau}},
$$

where the constants $t, \varsigma$ and $\tau$ depend on $L$ and $\Psi$ (see [10], Sect. 4.3, for details). Let us remark that (3.8) is in fact valid for a wide class of differential and integral operators. One key ingredient for the validation of (3.8) is the locality of the wavelets, see (2.21). Then, one can show ([10], Lem. 4.2 and Rem. 4.2) that for each $\lambda \in \nabla$ and a given tolerance $\varepsilon>0$ there exists a finite influence set $\mathcal{J}_{\lambda, \varepsilon} \subset \nabla$ such that the quantities

$$
e_{\lambda}:=\sum_{\lambda^{\prime} \in \Lambda \backslash \mathcal{J}_{\lambda, \varepsilon}}\left\langle L \psi_{\lambda^{\prime}}, \psi_{\lambda}\right\rangle x_{\lambda^{\prime}}, \quad x_{\lambda^{\prime}}:=\left\langle x_{\Lambda}, \tilde{\psi}_{\lambda^{\prime}}\right\rangle,
$$

satisfy

$$
\left(\sum_{\lambda \in \nabla \backslash \Lambda}\left|\gamma_{\lambda} e_{\lambda}\right|^{2}\right)^{1 / 2} \leq C_{\mathcal{J}} \varepsilon\left\|x_{\Lambda}\right\|_{H}
$$

for some constant $C_{\mathcal{J}}>0$.

Now, we may define the finite index set, which will reduce the infinite sum in (3.7). To be specific, let

$$
N_{\Lambda, \varepsilon}:=\left\{\lambda \in \nabla \backslash \Lambda: \Lambda \cap \mathcal{J}_{\lambda, \varepsilon} \neq \emptyset\right\}
$$

Thus, setting

$$
Z_{\Lambda}:=\left(\sum_{\lambda \in \nabla \backslash \Lambda}\left|\gamma_{\lambda} z_{\lambda}\right|^{2}\right)^{1 / 2}
$$

one can prove (see [10], Th. 4.1, and also [3]):

Proposition 3.2. Defining

$$
g_{\lambda}(\Lambda, \varepsilon):=\left|\gamma_{\lambda} \sum_{\lambda^{\prime} \in \Lambda \cap \mathcal{J}_{\lambda, \varepsilon}}\left\langle L \psi_{\lambda^{\prime}}, \psi_{\lambda}\right\rangle x_{\lambda^{\prime}}\right|, \quad \lambda \in \nabla \backslash \Lambda,
$$

the following estimates hold:

$$
\left\|x-x_{\Lambda}\right\|_{H} \leq C_{L} C_{\Psi}\left[\left(\sum_{\lambda \in N_{\Lambda, \varepsilon}} g_{\lambda}(\Lambda, \varepsilon)^{2}\right)^{1 / 2}+Z_{\Lambda}+\varepsilon C_{\mathcal{J}}\left\|x_{\Lambda}\right\|_{H}\right]
$$

and

$$
\left(\sum_{\lambda \in N_{\Lambda, \varepsilon}} g_{\lambda}(\Lambda, \varepsilon)^{2}\right)^{1 / 2} \leq \frac{1}{c_{L} c_{\Psi}}\left\|x-x_{\Lambda}\right\|_{H}+Z_{\Lambda}+\varepsilon C_{\mathcal{J}}\left\|x_{\Lambda}\right\|_{H}
$$

Now, (3.14) and (3.15) state that the sum over $g_{\lambda}(\Lambda, \varepsilon)$ is an efficient and reliable error estimator up to a fixed tolerance. On the other hand, in contrary to (3.7), it is now reduced to a finite sum over $N_{\Lambda, \varepsilon}$, so that it is in fact numerically accessible. 


\subsection{An adaptive refinement strategy}

Now, we may use the latter proposition to formulate a refinement strategy (see [10], Th. 4.2).

Proposition 3.3. Under the above assumptions, we have: Let eps $>0$ be a given tolerance and fix any $\vartheta^{*} \in$ $(0,1)$. Then, defining $C_{e}:=\frac{1}{c_{L} C_{\Psi}}+\frac{1-\vartheta^{*}}{2 C_{L} C_{\Psi}}$ and choosing $\mu^{*}>0$ such that $\mu^{*} C_{e} \leq \frac{1-\vartheta^{*}}{2\left(2-\vartheta^{*}\right) C_{L} C_{\Psi}}$, we set

$$
\varepsilon:=\frac{\mu^{*} \mathrm{eps}}{2 C_{\mathcal{J}}\left\|u_{\Lambda}\right\|_{H}}
$$

Suppose that $\Lambda \subset \nabla$ is chosen so that

$$
Z_{\Lambda}<\frac{1}{2} \mu^{*} \text { eps }
$$

Then whenever $\tilde{\Lambda} \subset \nabla, \Lambda \subset \tilde{\Lambda}$ is chosen so that

$$
\left(\sum_{\lambda \in \tilde{\Lambda} \cap N_{\Lambda, \varepsilon}} g_{\lambda}(\Lambda, \varepsilon)^{2}\right)^{1 / 2} \geq\left(1-\vartheta^{*}\right)\left(\sum_{\lambda \in N_{\Lambda, \varepsilon}} g_{\lambda}(\Lambda, \varepsilon)^{2}\right)^{1 / 2},
$$

there exists a constant $\kappa \in(0,1)$ such that either

$$
\left\|x_{\Lambda}-x_{\tilde{\Lambda}}\right\|_{H} \geq \kappa\left\|x-x_{\Lambda}\right\|_{H}
$$

or

$$
\left(\sum_{\lambda \in N_{\Lambda, \varepsilon}} g_{\lambda}(\Lambda, \varepsilon)^{2}\right)^{1 / 2}=\left(\sum_{\lambda \in \nabla \backslash \Lambda} g_{\lambda}(\Lambda, \varepsilon)^{2}\right)^{1 / 2}<\text { eps. }
$$

In [10], the distance property (3.19) is used to prove the convergence of the above strategy for symmetric positive definite operators (see also Sect. 4 below). In our general setting, a convergence result can not be expected.

\subsection{Saddle point problems}

Now, we apply the above results to the saddle point operator $\mathcal{A}$ in (2.6). Obviously, assumption (3.1) is fulfilled by (2.7). As a wavelet basis on $H=\mathcal{H}$, we choose $\Psi \times \Theta$ and then (2.23) and (2.24) imply (3.3) for the weight factors

$$
\gamma_{[\lambda, \mu]}:=\left(\begin{array}{c}
2^{-t|\lambda|} \\
2^{-s|\mu|}
\end{array}\right), \quad[\lambda, \mu] \in \nabla^{X} \times \nabla^{M},
$$

and $y=[v, q] \in X \times M=H$. In fact, for $\left[v^{\prime}, q^{\prime}\right] \in X^{\prime} \times M^{\prime}$, we have

$$
\begin{aligned}
\left\|\left[v^{\prime}, q^{\prime}\right]\right\|_{H^{\prime}}^{2} & =\left\|v^{\prime}\right\|_{X^{\prime}}^{2}+\left\|q^{\prime}\right\|_{M^{\prime}}^{2} \\
& \sim \sum_{\lambda \in \nabla^{X}} 2^{-2 t|\lambda|}\left|\left\langle v^{\prime}, \psi_{\lambda}\right\rangle_{X^{\prime} \times X}\right|^{2}+\sum_{\mu \in \nabla^{M}} 2^{-2 s|\mu|}\left|\left\langle q^{\prime}, \vartheta_{\mu}\right\rangle_{M^{\prime} \times M}\right|^{2} \\
& =\sum_{[\lambda, \mu] \in \nabla^{X} \times \nabla^{M}}\left|\left(\begin{array}{c}
2^{-t|\lambda|}\left\langle v^{\prime}, \psi_{\lambda}\right\rangle_{X^{\prime} \times X} \\
2^{-s|\mu|}\left\langle q^{\prime}, \vartheta_{\mu}\right\rangle_{M^{\prime} \times M}
\end{array}\right)\right|^{2} \\
& =\sum_{[\lambda, \mu] \in \nabla^{X} \times \nabla^{M}}\left|\gamma_{[\lambda, \mu]}\left\langle\left[v^{\prime}, q^{\prime}\right],\left[\psi_{\lambda}, \vartheta_{\mu}\right]\right\rangle_{\mathcal{H}^{\prime} \times \mathcal{H}}\right|^{2} .
\end{aligned}
$$


Now, we define the residual

$$
R_{\Lambda}:=\left(\begin{array}{c}
r_{\Lambda} \\
\rho_{\Lambda}
\end{array}\right):=\mathcal{A}\left(\begin{array}{c}
u_{\Lambda}-u \\
p_{\Lambda}-p
\end{array}\right)=\left(\begin{array}{c}
A u_{\Lambda}+B^{\prime} p_{\Lambda}-f \\
B u_{\Lambda}-g
\end{array}\right)
$$

and the quantities

$$
\varrho_{\lambda}:=\left|\left\langle r_{\Lambda}, \psi_{\lambda}\right\rangle_{X^{\prime} \times X}\right|, \quad \lambda \in \nabla^{X}, \quad \zeta_{\mu}:=\left|\left\langle\rho_{\Lambda}, \vartheta_{\mu}\right\rangle_{M^{\prime} \times M}\right|, \quad \mu \in \nabla^{M} .
$$

Theorem 3.4. For the above discretization $\left(X_{\Lambda}, M_{\Lambda}\right)$, the following error estimate is valid:

$$
\left\|u-u_{\Lambda}\right\|_{X}+\left\|p-p_{\Lambda}\right\|_{M} \sim\left(\sum_{\lambda \in \nabla^{X} \backslash \Lambda^{X}} 2^{-2 t|\lambda|} \varrho_{\lambda}^{2}\right)^{1 / 2}+\left(\sum_{\mu \in \nabla^{M} \backslash \Lambda^{M}} 2^{-2 s|\mu|} \zeta_{\mu}^{2}\right)^{1 / 2} .
$$

As a consequence, we get the following result:

Corollary 3.5. For equilibrated discretizations, the following equivalence holds for $g \equiv 0$ :

$$
\left\|u-u_{\Lambda}\right\|_{X} \sim\left(\sum_{\lambda \in \nabla^{X} \backslash \Lambda^{X}} 2^{-2 t|\lambda|} \varrho_{\lambda}^{2}\right)^{1 / 2}
$$

Remark 3.6. In view of Proposition 2.1, the estimates (3.23), (3.24) also hold if we replace $\left\|u-u_{\Lambda}\right\|_{X}+\| p-$ $p_{\Lambda} \|_{M}$ by the energy norm $\left\|\left[u-u_{\Lambda}, p-p_{\Lambda}\right]\right\|_{\mathcal{A}}$ and $\left\|u-u_{\Lambda}\right\|_{X}$ by $\left\|u-u_{\Lambda}\right\|_{A}$, respectively, provided that the assumptions of Proposition 2.1 are fulfilled.

Finally, we apply the results concerning the adaptive strategy to saddle point problems. Firstly, we have to assume that both $A$ and $B$ are quasi sparse in the sense of (3.8), where for $B$ we have to replace $t$ in (3.8) by $s$. Then, for $u_{\lambda^{\prime}}:=\left\langle u, \tilde{\psi}_{\lambda^{\prime}}\right\rangle_{X^{\prime} \times X}, \lambda^{\prime} \in \nabla^{X}$, and $p_{\mu^{\prime}}:=\left\langle p, \tilde{\vartheta}_{\mu^{\prime}}\right\rangle_{M^{\prime} \times M}, \mu^{\prime} \in \nabla^{M}$, the quantity in (3.9) becomes

$$
e_{[\lambda, \mu]}=\sum_{\substack{\left[\lambda^{\prime}, \mu^{\prime}\right] \in \\
\left(\Lambda^{X} \backslash \mathcal{J}_{\lambda, \varepsilon}^{X}\right) \times\left(\Lambda^{M} \backslash \mathcal{J}_{\mu, \varepsilon}^{M}\right)}}\left(\begin{array}{c}
u_{\lambda^{\prime}}\left\langle A \psi_{\lambda^{\prime}}, \psi_{\lambda}\right\rangle_{X^{\prime} \times X}+p_{\mu^{\prime}}\left\langle B^{\prime} \vartheta_{\mu^{\prime}}, \psi_{\lambda}\right\rangle_{X^{\prime} \times X} \\
u_{\lambda^{\prime}}\left\langle B \psi_{\lambda^{\prime}}, \vartheta_{\mu}\right\rangle_{M^{\prime} \times M}
\end{array}\right),
$$

where $\mathcal{J}_{\lambda, \varepsilon}^{X} \subset \nabla^{X}$ and $\mathcal{J}_{\mu, \varepsilon}^{M} \subset \nabla^{M}$ are suitable finite influence sets. Next, setting $f_{\lambda}:=\left\langle f, \psi_{\lambda}\right\rangle_{X^{\prime} \times X}, \lambda \in \nabla^{X}$ and $g_{\mu}:=\left\langle g, \vartheta_{\mu}\right\rangle_{M^{\prime} \times M}, \mu \in \nabla^{M}$, equation (3.12) reads

$$
Z_{\Lambda}=\left(\sum_{[\lambda, \mu] \in\left(\nabla^{X} \backslash \Lambda^{X}\right) \times\left(\nabla^{M} \backslash \Lambda^{M}\right)} 2^{-2 t|\lambda|}\left|f_{\lambda}\right|^{2}+2^{-2 s|\mu|}\left|g_{\mu}\right|^{2}\right)^{1 / 2}
$$

Finally, the error quantities $g_{[\lambda, \mu]}(\Lambda, \varepsilon)$ defined in (3.13) take the form

$$
\begin{array}{r}
g_{[\lambda, \mu]}(\Lambda, \varepsilon)=\mid \sum_{\left[\lambda^{\prime}, \mu^{\prime}\right] \in\left(\Lambda^{X} \cap \mathcal{J}_{\lambda, \varepsilon}^{X}\right) \times\left(\Lambda^{M} \cap \mathcal{J}_{\mu, \varepsilon}^{M}\right)}\left(2^{-t|\lambda|} u_{\lambda^{\prime}}\left\langle A \psi_{\lambda^{\prime}}, \psi_{\lambda}\right\rangle_{X^{\prime} \times X}\right. \\
\left.+2^{-t|\lambda|} p_{\mu^{\prime}}\left\langle B^{\prime} \vartheta_{\mu^{\prime}}, \psi_{\lambda}\right\rangle_{X^{\prime} \times X}+2^{-s|\mu|} u_{\lambda^{\prime}}\left\langle B \psi_{\lambda^{\prime}}, \vartheta_{\mu}\right\rangle_{M^{\prime} \times M}\right) \mid .
\end{array}
$$

With these definitions, Proposition 3.3 easily applies. 


\section{Convergence of ADAPtive schemes}

So far, we have set up an a posteriori error analysis for adaptively refined wavelet spaces. However, it remains to study the convergence of such an adaptive algorithm. In [10], the above described adaptive wavelet strategy for positive definite operators was proven to converge. Now, one might think that the generalization to saddle point problems is an easy task.

Unfortunately, we did not succeed in adapting the arguments used in [10] to saddle point problems. Let us briefly point out the main differences when going from a positive definite to an indefinite problem. We consider the Problem $A u=f$, where $A: H_{0}^{t}(\Omega) \rightarrow H^{-t}(\Omega)$ is some positive definite, boundedly invertible operator and $f \in H^{-t}(\Omega)$ are the given data, while the function $u \in H_{0}^{t}(\Omega)$ has to be seeked. Taking as above a wavelet basis $\Psi=\left\{\psi_{\lambda}: \lambda \in \nabla\right\} \subset H_{0}^{t}(\Omega)$, we denote by $u_{\Lambda}$ the Galerkin solution w.r.t. a (finite) set $\Lambda \subset \nabla$.

In the previous section, we have described a strategy how to enlarge $\Lambda$ to some $\tilde{\Lambda} \supset \Lambda$ such that the distance property holds, i.e., there exists some $0<\kappa<1$ such that

$$
\left\|u_{\Lambda}-u_{\tilde{\Lambda}}\right\|_{A} \geq \kappa\left\|u-u_{\Lambda}\right\|_{A}
$$

see Proposition 3.3, (3.19). Note that here the energy norm $\|\cdot\|_{A}$ is used, which already assumes that $A$ is positive definite. Now, one proceeds using Galerkin orthogonality

$$
a\left(u_{\Lambda}-u_{\tilde{\Lambda}}, u-u_{\tilde{\Lambda}}\right)=0
$$

to conclude

$$
\left\|u-u_{\tilde{\Lambda}}\right\|_{A}^{2}=\left\|u-u_{\Lambda}\right\|_{A}^{2}-\left\|u_{\Lambda}-u_{\tilde{\Lambda}}\right\|_{A}^{2} \leq\left(1-\kappa^{2}\right)\left\|u-u_{\Lambda}\right\|_{A}^{2},
$$

which proves the saturation property, i.e., a strict error reduction since $0<1-\kappa^{2}<1$.

Unfortunately, (4.1) is no longer true when $A$ is replaced by the operator $\mathcal{A}$ in (2.6) which represents a saddle point operator. Now, one could try to use that $\mathcal{A}$ is positive definite on $V=\operatorname{Ker}(B)$. This approach in fact gives rise to a convergent adaptive algorithm (which can be numerically performed) for computing $u$ provided that a basis for $V$ is explicitly available, [12]. This of course contradicts the philosophy of the saddle point approach and is not what we aim at.

As a second approach, one could consider the reduced problem for $p$, i.e.,

$$
S p=B A^{-1} f-g,
$$

involving the Schur complement $S$. But also this approach seems to have some ultimate obstacles. Firstly, due to the presence of $A^{-1}$, the entries of the corresponding stiffness matrix $\left\langle S \vartheta_{\mu}, \vartheta_{\mu^{\prime}}\right\rangle_{M^{\prime} \times M}$ can not be easily computed (and the same is true for the right-hand side). Now, one could approximate $A^{-1}$ by some $A_{\Lambda}^{-1}$. But then one ends up with the problem that the discretization of $S$ is not the same as discretizing the three factors separately, i.e.,

$$
B_{\Lambda} A_{\Lambda}^{-1} B_{\Lambda}^{\prime} \neq S_{\Lambda}
$$

This means that the computed solution does not correspond to the Galerkin solution w.r.t. $\Lambda$. This, however, is essential for (4.1). Note that the non-equality in (4.2) still holds if one could replace $B_{\Lambda}, B_{\Lambda}^{\prime}$ by $B$ and $B^{\prime}$, respectively. In Section 5 below we will describe situations where this is in fact possible.

Next, one could try to study the error between the real Galerkin solution $p_{\Lambda}$ w.r.t. $S_{\Lambda}$ and the perturbed one $p_{\Lambda}^{*}$ w.r.t. $B_{\Lambda} A_{\Lambda}^{-1} B_{\Lambda}^{\prime}$ (which is available for example by the Uzawa algorithm). We have not been able to give meaningful quantitative criteria for the index set $\Lambda$ such that this error is below some given tolerance.

Hence, we have been looking for a new approach that circumvents all the above listed problems and drawbacks. In this section, we introduce an alternative, namely an adaptive version of Uzawa's algorithm. The analysis of 
this method leads us to the desired result, namely a convergent adaptive refinement strategy for saddle point problems.

\subsection{An adaptive Uzawa algorithm}

The Uzawa algorithm is a well-known iterative solver for saddle point problems, [2]. We aim at using this algorithm as an outer iteration for an adaptive method. To this end, we consider the Uzawa algorithm for (infinite dimensional) Hilbert spaces. In a second step, we formulate our adaptive version of Uzawa's algorithm. This adaptive version creates some additional errors that need to be controlled over the iteration.

\subsubsection{Uzawa algorithm in Hilbert spaces}

Originally, the Uzawa algorithm was formulated for saddle point problems involving matrices of finite dimension, [2]. Here, we consider its formulation in infinite dimensional Hilbert spaces $X$ and $M$. Given any bounded linear operator $R: M^{\prime} \rightarrow M$ (whose role will be discussed later) and $\alpha \in \mathbb{R}$, we consider the following variant of the Uzawa algorithm:

Algorithm 4.1. Given any $p^{(0)} \in M$, we compute $u^{(i)}$ and $p^{(i)}$ for $i=1,2, \ldots$, by

$$
\begin{aligned}
A u^{(i)} & =f-B^{\prime} p^{(i-1)}, \\
p^{(i)} & =p^{(i-1)}+\alpha R B u^{(i)} .
\end{aligned}
$$

The convergence of this algorithm is well-known if $R$ is the Riesz operator (see [7] and the references therein). However, since for the subsequent error analysis it will be important to keep track of the influence of the data to the error, we need an explicit error estimate here. Hence, we state the following result and include also the proof for completeness and convenience.

Theorem 4.2. Let $A$ be elliptic on all of $X$ and suppose that $R S$ is selfadjoint and positive definite and $0<\alpha<2\|R S\|_{[M]}^{-1}$ (S again being the Schur complement). Then the Algorithm 4.1 converges. To be precise, for $p^{(0)}:=0$ and setting $q:=\|I d-\alpha R S\|_{[M]}$, we obtain $q<1$ and the following error estimate

$$
\left\|p-p^{(i)}\right\|_{M} \leq\left\|A^{-1} f\right\|_{X}\|\alpha R B\|_{[X, M]} \frac{q^{i}}{1-q}
$$

Proof. By induction, it is easy to show that

$$
p^{(i)}=(I d-\alpha R S)^{i} p^{(0)}+\left(\sum_{k=0}^{i-1}(I d-\alpha R S)^{k}\right) \alpha R B A^{-1} f .
$$

Now, let $H$ be a Hilbert space. Then, for any linear bounded and selfadjoint operator $T: H \rightarrow H$, the following equation is well-known [1]

$$
\sup \{|\lambda|: \lambda \in \sigma(T)\}=\|T\|_{[H]},
$$

where the norm is the operator norm induced by the norm in $H$ and $\sigma(T)$ denotes the spectrum of $T$. Now, we use the fact that $R S$ is selfadjoint and hence

$$
\sup \{|\lambda|: \lambda \in \sigma(\alpha R S)\}=\|\alpha R S\|_{[M]}=\alpha\|R S\|_{[M]}<2 .
$$

The assumptions on $\alpha$ and on $R S$ imply that $\sigma(I d-\alpha R S) \subset(-1,1)$, since

$$
\sup \{\lambda: \lambda \in \sigma(I d-\alpha R S)\}=1-\alpha \inf \{\lambda: \lambda \in \sigma(R S)\}<1
$$


and

$$
\begin{aligned}
\inf \{\lambda: \lambda \in \sigma(I d-\alpha R S)\} & =1-\alpha \sup \{|\lambda|: \lambda \in \sigma(R S)\} \\
& =1-\alpha\|R S\|_{[M]}>-1 .
\end{aligned}
$$

Consequently, we obtain

$$
q=\|I d-\alpha R S\|_{[M]}=\sup \{|\lambda|: \lambda \in \sigma(I d-\alpha R S)\}<1 .
$$

This finally implies, using $q<1$ and $p^{(0)}=0$

$$
\begin{aligned}
\left\|p-p^{(i)}\right\|_{M} & =\left\|S^{-1} B A^{-1} f-\sum_{k=0}^{i-1}(I d-\alpha R S)^{k} \alpha R B A^{-1} f\right\|_{M} \\
& \leq\left\|A^{-1} f\right\|_{X}\left\|\sum_{k=i}^{\infty}(I d-\alpha R S)^{k} \alpha R B\right\|_{[X, M]} \\
& \leq\left\|A^{-1} f\right\|_{X}\|\alpha R B\|_{[X, M]} \frac{q^{i}}{1-q},
\end{aligned}
$$

which proves (4.5).

Let us add some comments on the role of the operator $R$ in (4.4). One natural choice is the Riesz operator. However, we do not want to restrict Algorithm 4.1 to this case only.

\subsubsection{Adaptive version}

Now, in general, we cannot compute $u^{(i)}$ and $p^{(i)}$ in each step exactly but only with some approximations. Note that $u^{(i)}$ and $p^{(i)}$ are elements of infinite dimensional spaces. We in fact compute approximations $u_{\Lambda}^{(i)}, p_{\Lambda}^{(i)}$ with respect to finite dimensional subsets $\Lambda_{i}=\left(\Lambda_{i}^{X}, \Lambda_{i}^{M}\right) \subset \nabla^{X} \times \nabla^{M}$. The aim of this subsection is to study the overall error in the Uzawa iteration introduced by this approximation, where $\Lambda_{i}$ will be chosen adaptively.

To be precise, we define $\tilde{u}^{(i)}$ as the solution of

$$
A \tilde{u}^{(i)}=f-B^{\prime} p_{\Lambda}^{(i-1)}
$$

(which is not computable) and we assume that we approximate $\tilde{u}^{(i)}$ by the Galerkin solution $u_{\Lambda}^{(i)}$ of (4.9) with respect to an adaptively created index set $\Lambda_{i}^{X} \subset \nabla^{X}$ up to a certain error, i.e.,

$$
\left\|u_{\Lambda}^{(i)}-\tilde{u}^{(i)}\right\|_{X}<q^{i} \varepsilon_{i}
$$

where we may choose $\varepsilon_{i}$. Now, we can formulate our adaptive Uzawa iteration:

Algorithm 4.3. Let $\Lambda_{0}^{M}=\emptyset$ and $p_{\Lambda_{0}}^{(0)}=p^{(0)}=0$. Then, for $i=1,2, \ldots$, and given $\Lambda_{i-1}^{M}$, proceed as follows:

1. Use a convergent adaptive algorithm to solve (4.9), i.e., determine a set of indices $\Lambda_{i}^{X}$ and the corresponding Galerkin solution $u_{\Lambda}^{(i)}$ such that (4.10) holds.

2. Determine an index set $\Lambda_{i}^{M}$ such that $R B\left(X_{\Lambda_{i}}\right) \subseteq M_{\Lambda_{i}}$.

3. Set $p_{\Lambda}^{(i)}:=p_{\Lambda}^{(i-1)}+\alpha R B u_{\Lambda}^{(i)}$.

In Section 5 below, we will investigate the meaning of the condition $R B\left(X_{\Lambda_{i}}\right) \subseteq M_{\Lambda_{i}}$ in more detail. Let us now study the convergence of Algorithm 4.3. 
Theorem 4.4. Let $A$ be elliptic on all of $X$ and assume that $\varepsilon:=\left\{\varepsilon_{i}\right\}_{i=0}^{\infty} \in \ell_{1}\left(\mathbb{N}_{0}\right)$. Then, we have

$$
\left\|p^{(i)}-p_{\Lambda}^{(i)}\right\|_{M} \leq\|\varepsilon\|_{\ell_{1}\left(\mathbb{N}_{0}\right)}\|\alpha R B\|_{[X, M]} q^{i},
$$

where $p^{(0)}=p_{\Lambda_{0}}^{(0)}$ and $p^{(i)}$ is defined by (4.6).

Proof. As above, it is readily seen that

$$
p^{(i)}-p_{\Lambda}^{(i)}=(I d-\alpha R S)\left(p^{(i-1)}-p_{\Lambda}^{(i-1)}\right)-\alpha R B\left(u_{\Lambda}^{(i)}-\tilde{u}^{(i)}\right) .
$$

By iteration and assuming that $p^{(0)}=p_{\Lambda_{0}}^{(0)}$, we obtain

$$
p_{\Lambda}^{(i)}-p^{(i)}=\sum_{k=0}^{i-1}(I d-\alpha R S)^{k} \alpha R B\left(u_{\Lambda}^{(i-k)}-\tilde{u}^{(i-k)}\right) .
$$

Inserting our assumption (4.10), we conclude that

$$
\left\|p^{(i)}-p_{\Lambda}^{(i)}\right\|_{M} \leq\|\alpha R B\|_{[X, M]} q^{i} \sum_{k=0}^{i-1} \varepsilon_{k} \leq\|\varepsilon\|_{\ell_{1}\left(\mathbb{N}_{0}\right)}\|\alpha R B\|_{[X, M]} q^{i},
$$

which proves the result.

Finally, we obtain our desired result:

Theorem 4.5. Under the above assumptions, we obtain the following error estimates for the adaptive Uzawa Algorithm 4.3:

(a) The Algorithm 4.3 converges, i.e., we have

$$
\left\|p-p_{\Lambda}^{(i)}\right\|_{M} \lesssim q^{i} .
$$

(b) The solution of the saddle point problem can be approximated with any desired accuracy:

$$
\left\|u-u_{\Lambda}^{(i+1)}\right\|_{X}+\left\|p-p_{\Lambda}^{(i)}\right\|_{M} \lesssim q^{i}
$$

Proof. Using the triangle inequality and the Theorems 4.2 and 4.4 gives

$$
\begin{aligned}
\left\|p-p_{\Lambda}^{(i)}\right\|_{M} & \leq\left\|p-p^{(i)}\right\|_{M}+\left\|p^{(i)}-p_{\Lambda}^{(i)}\right\|_{M} \\
& \leq\left\|A^{-1} f\right\|_{X}\|\alpha R B\|_{[X, M]} \frac{q^{i}}{1-q}+C\|\alpha R B\|_{[X, M]} q^{i} \\
& =q^{i}\|\alpha R B\|_{[X, M]}\left(\left\|A^{-1} f\right\|_{X} \frac{1}{1-q}+C\right),
\end{aligned}
$$

which proves part (a) of the claim. For proving (b), we use standard arguments to obtain

$$
\left\|u-\tilde{u}^{(i+1)}\right\|_{X} \lesssim\left\|B^{\prime} p_{\Lambda}^{(i)}-B^{\prime} p\right\|_{X^{\prime}} \lesssim q^{i},
$$

where we have used (a) in the last step. Finally, using triangle inequality and (4.10) yields

$$
\left\|u-u_{\Lambda}^{(i+1)}\right\|_{X} \leq\left\|u-\tilde{u}^{(i+1)}\right\|_{X}+\left\|\tilde{u}^{(i+1)}-u_{\Lambda}^{(i+1)}\right\|_{X} \lesssim q^{i},
$$

which proves the desired result. 
Remark 4.6. It is remarkable that the above convergence analysis does not require the validity of the LBB condition. The spaces $X_{\Lambda_{i}}$ and $M_{\Lambda_{i}}$ are only linked through the (weak) condition $R B\left(X_{\Lambda_{i}}\right) \subseteq M_{\Lambda_{i}}$ which will be investigated in more detail in the subsequent section.

One may interpret the fact that no LBB condition is required in the following way. In the classical sense, (LBB) is a condition for a linear approximation process when the dimension of the trial spaces grows. Here, the nonlinear approximation process formed by the adaptive method automatically picks the appropriate basis functions.

\subsection{Approximate application of $B$ and $B^{\prime}$}

The presented algorithm in the latter section relies on the fact that the application of the operators $B$ and $B^{\prime}$ can be performed exactly on the finite-dimensional trial spaces. In the next section, we will give explicit criteria that show under which circumstances this framework is in fact feasible. However, sometimes one may be interested in a somewhat more general situation where only approximations are available. In this section, we will describe and analyze a corresponding variant of our algorithm.

To be precise, we will pose the following assumptions on the approximations:

\section{Assumption 4.7.}

1. Let a finite set $\Lambda^{X} \subset \nabla^{X}$ and $u_{\Lambda} \in X_{\Lambda^{X}}$ are given. Then, for any $\tau>0$ there exists an index set $\tilde{\Lambda}^{M}(\tau) \subset \nabla^{M}$ and a computable approximation $(R B)_{\tau} u_{\Lambda} \in M_{\tilde{\Lambda}^{M}(\tau)}$ such that

$$
\left\|\left[(R B)_{\tau}-R B\right] u_{\Lambda}\right\|_{M}<\tau
$$

2. Let a finite set $\Lambda^{M} \subset \nabla^{M}$ and $p_{\Lambda} \in M_{\Lambda^{M}}$ are given. Then, for any $\delta>0$ there exists an index set $\tilde{\Lambda}^{X}(\delta) \subset \nabla^{X}$ and a computable approximation $B_{\delta}^{\prime} p_{\Lambda} \in \tilde{X}_{\tilde{\Lambda}^{X}(\delta)}$ such that

$$
\left\|\left(B_{\delta}^{\prime}-B^{\prime}\right) p_{\Lambda}\right\|_{X^{\prime}}<\delta
$$

In [9], an adaptive approximate application of elliptic operators in terms of wavelet bases has been introduced. This can also be generalized to our framework. Hence, the above assumptions are in fact reasonable. Under this assumption, we formulate the following:

Algorithm 4.8. Let $\Lambda_{0}^{M}=\emptyset$ and $p_{\Lambda_{0}}^{(0)}=p^{(0)}=0$. Then, for $i=1,2, \ldots$, and given $\Lambda_{i-1}^{M}$ fix parameters $\delta_{i}, \tau_{i}$ and proceed as follows:

1. Use a convergent adaptive algorithm in order to solve

$$
A \bar{u}^{(i)}=f-B_{\delta_{i}}^{\prime} p_{\Lambda}^{(i-1)}
$$

i.e., determine an index set $\Lambda_{i}^{X} \subset \mathcal{J}^{X}$ and the corresponding Galerkin solution $u_{\Lambda}^{(i)}$ of (4.14) such that

$$
\left\|u_{\Lambda}^{(i)}-\bar{u}^{(i)}\right\|_{X}<q^{i} \varepsilon_{i}\left(3\|\alpha R B\|_{[X, M]}\right)^{-1}
$$

2. $\operatorname{Set} \Lambda_{i}^{M}:=\Lambda_{i-1}^{M} \cup \tilde{\Lambda}^{M}\left(\tau_{i}\right)$ and $p_{\Lambda}^{(i)}:=p_{\Lambda}^{(i-1)}+\alpha(R B)_{\tau_{i}} u_{\Lambda}^{(i)}$.

The convergence of Algorithm 4.8 is given by the following result:

Theorem 4.9. Under the above assumption we have

$$
\left\|p^{(i)}-p_{\Lambda}^{(i)}\right\|_{M} \leq\|\varepsilon\|_{\ell_{1}\left(\mathbb{N}_{0}\right)} q^{i}
$$


where $p^{(0)}=p_{\Lambda_{0}}^{(0)}$ and the parameters are set according to

$$
\delta_{i}:=\left(\left\|A^{-1}\right\|_{\left[X^{\prime}, X\right]}\|\alpha R B\|_{[X, M]}\right)^{-1} \frac{1}{3} q^{i} \varepsilon_{i}, \quad \tau_{i}:=\frac{1}{3 \alpha} q^{i} \varepsilon_{i} .
$$

Proof. We need to consider the auxilliary quantity $\check{u}^{(i)}$ which is defined as the solution of

$$
A \check{u}^{(i)}=f-B^{\prime} p_{\Lambda}^{(i-1)} .
$$

Then, by similar techniques as in the proofs of the above theorems, we obtain

$$
p_{\Lambda}^{(i)}-p^{(i)}=\sum_{k=0}^{i-1} \alpha(I d-\alpha R S)^{k}\left(R B\left(\check{u}^{(i-k)}-u_{\Lambda}^{(i-k)}\right)+\left[R B-(R B)_{\tau_{i-k}}\right] u_{\Lambda}^{(i-k)}\right) .
$$

Using the estimate

$$
\begin{aligned}
\left\|\check{u}^{(i)}-u_{\Lambda}^{(i)}\right\|_{X} & \leq\left\|A^{-1}\right\|_{\left[X^{\prime}, X\right]}\left\|\left(B_{\delta_{i}}^{\prime}-B^{\prime}\right) p_{\Lambda}^{(i-1)}\right\|_{X}+\left\|\bar{u}^{(i)}-u_{\Lambda}^{(i)}\right\|_{X} \\
& <\frac{2}{3 \alpha}\|R B\|_{[X, M]}^{-1} q^{i} \varepsilon_{i}
\end{aligned}
$$

and the definition of the parameters gives the desired result.

Corollary 4.10. Under the above assumptions, the following estimate holds

$$
\left\|u-u_{\Lambda}^{(i+1)}\right\|_{X}+\left\|p-p_{\Lambda}^{(i)}\right\|_{M} \lesssim q^{i}
$$

Now, several remarks on the above results are in order:

- As can be seen, a convergent adaptive strategy for the positive definite operator $A$ builds the basic kernel of our method. By assuming that this algorithm reduces the error to $q^{i} \varepsilon_{i}$, we implicitly assumed the convergence of the inner iteration, i.e., we assume that there exists a strategy to build $\Lambda_{i}^{X}$ which allows this error reduction. As already pointed out, the algorithm in [10] meets this requirement.

Also the question arises how large the set $\Lambda_{i}^{X}$ is, i.e., how many degrees of freedom are necessary to reach the desired accuracy. This is a property of the adaptive strategy used for step 1 in Algorithm 4.3 and the possible fill-ins due to the second step of our Uzawa algorithm. For example, the method introduced in [9] for positive definite operators was proven to have asymptotically optimal complexity. However, we will not study the complexity of our adaptive Uzawa algorithm here.

- Clearly, the essential quantity $q=\|I d-\alpha R S\|_{[X, M]}<1$ determining the speed of convergence will often not be available exactly. One could however estimate $q$ in order to obtain a priori a maximum number of (outer) Uzawa iterations to reach the desired accuracy. Of course, an estimate for $q$ depends on the various data for a particular saddle point problem.

- At a first look one might get the impression that the performance of Algorithm 4.3 depends only on $p$ whereas the choice of the adaptive index sets $\Lambda_{i}$ depends only on $u$. However, the situation is somewhat more involved. Since the behaviour of the right-hand side influences the choice of $\Lambda_{i}$, it can be seen that $p$ in fact effects the adaptive refinement. On the other hand, $u$ influences also the Uzawa algorithm since $u^{(i)}$ enters the definition of $p^{(i)}$.

- Finally, we comment on the relationship of the above algorithm to the inexact Uzawa algorithm. The latter one has recently been studied e.g. in [6,17], where an error analysis is given if the elliptic subproblem corresponding to (4.3) is only solved up to some tolerance (in this sense inexact). Moreover, the preconditioning of this method is considered there. One might think that our algorithm is simply a variant of such an inexact Uzawa iteration. However, again, we point out that our method works in infinite dimensional Hilbert spaces and the error analysis considers the inexact solution of the continuous elliptic problem in (4.3). To our knowledge, inexact Uzawa iterations are based on finite dimensional spaces. 


\section{Multiscale Bases, the LBB and FEP COndition}

Even though (LBB) and (FEP) are not directly needed for proving the convergence of the Algorithm 4.3 and 4.8 above, these properties play a key role for the analysis of discretizations of saddle point problems. Moreover, it will turn out that these properties are closely linked to situations where an exact evaluation of the operators $B$ and $B^{\prime}$ on the trial spaces is possible. Hence, this section is devoted to conditions on the particular choice of multiscale trial spaces in order to fulfill (LBB) and (FEP). It will turn out that biorthogonality is the main technical tool to derive explicit criteria.

\subsection{The LBB condition}

The LBB condition has already been studied in the wavelet context in [4,16,21]. All papers are however restricted to the Stokes problem. While [16] does not consider adaptively refined spaces, in [4] this problem is treated using ideas from [16]. But the conditions derived in [4] are still somewhat implicit since certain integrals have to be checked. Here, we will deal with arbitrary saddle point problems and we derive explicit criteria for the adaptively refined spaces in order to fulfill (LBB). By "explicit", we mean that given one of the two discretization spaces, the missing one can directly be constructed by only looking at the wavelet indices without further checks. The basic idea, namely to use biorthogonality and the following well-known result by M. Fortin can already be found in $[16]$.

Proposition 5.1. ([18]) The $L B B$ condition holds if and only if there exists an operator $Q_{\Lambda} \in \mathcal{L}\left(X, X_{\Lambda}\right)$ satisfying

$$
\begin{aligned}
b\left(v-Q_{\Lambda} v, q_{\Lambda}\right) & =0 \text { for all } v \in X, q_{\Lambda} \in M_{\Lambda}, \text { and } \\
\left\|Q_{\Lambda}\right\|_{\mathcal{L}(X, X)} & \lesssim 1,
\end{aligned}
$$

independent of $\Lambda$.

For the spaces $X_{\Lambda}$ defined above, we consider the operator $Q_{\Lambda}$ given by

$$
Q_{\Lambda} v:=\sum_{\lambda \in \Lambda^{X}}\left\langle v, \tilde{\psi}_{\lambda}\right\rangle_{X \times X^{\prime}} \psi_{\lambda}
$$

Due to the norm equivalences (2.23), condition (5.2) is always fulfilled:

$$
\left\|Q_{\Lambda} v\right\|_{X}^{2} \sim \sum_{\lambda \in \Lambda^{X}} 2^{2 t|\lambda|}\left|\left\langle v, \tilde{\psi}_{\lambda}\right\rangle_{X \times X^{\prime}}\right|^{2} \leq \sum_{\lambda \in \nabla} 2^{2 t|\lambda|}\left|\left\langle v, \tilde{\psi}_{\lambda}\right\rangle_{X \times X^{\prime}}\right|^{2} \lesssim\|v\|_{X}^{2} .
$$

For any subset $\bar{X} \subseteq X$ we will use the notations

$$
\bar{X}^{\perp_{b}}:=\{q \in M: b(v, q)=0 \text { for all } v \in \bar{X}\},
$$

and similar for $\bar{M} \subseteq M$

$$
\bar{M}^{\perp_{b}}:=\{v \in X: b(v, q)=0 \text { for all } q \in \bar{M}\} .
$$

Moreover, we use the standard definition of the polar space for any subset $\bar{X} \subseteq X$

$$
\bar{X}^{0}:=\left\{x^{\prime} \in X^{\prime}:\left\langle x^{\prime}, v\right\rangle_{X^{\prime} \times X}=0 \text { for all } v \in \bar{X}\right\},
$$

and similarly for subsets in $M$. By definition, we have

$$
X_{\Lambda}^{\perp_{b}}=B\left(X_{\Lambda}\right)^{0} \quad \text { and } \quad M_{\Lambda}^{\perp_{b}}=B^{\prime}\left(M_{\Lambda}\right)^{0} .
$$


Now, we obtain the desired result.

Theorem 5.2. The multiscale spaces $X_{\Lambda}, M_{\Lambda}$ defined above fulfill the LBB condition (2.25) provided that one of the following equivalent conditions holds:

(a) $M_{\Lambda} \subseteq\left(X \ominus X_{\Lambda}\right)^{\perp_{b}}$,

(b) $B^{\prime}\left(M_{\Lambda}\right) \subseteq \tilde{X}_{\Lambda}$,

(c) $B\left(X \ominus X_{\Lambda}\right) \subseteq M^{\prime} \ominus \tilde{M}_{\Lambda}$.

Proof. Due to the Riesz basis property, we have for $v \in X$ and $q_{\Lambda} \in M_{\Lambda}$

$$
b\left(v-Q_{\Lambda} v, q_{\Lambda}\right)=\sum_{\lambda \in \nabla \backslash \Lambda}\left\langle v, \tilde{\psi}_{\lambda}\right\rangle_{X \times X^{\prime}} b\left(\psi_{\lambda}, q_{\Lambda}\right)=0
$$

if and only if

$$
b\left(v_{\mu}, q_{\Lambda}\right)=0 \quad \text { for all } v_{\mu} \in X \ominus X_{\Lambda}, q_{\Lambda} \in M_{\Lambda},
$$

which is equivalent to (a). It remains to verify the equivalence of (a)-(c). In fact, using $M_{\Lambda}^{0}=M^{\prime} \ominus \tilde{M}_{\Lambda}$ and $\tilde{X}_{\Lambda}^{0}=X \ominus X_{\Lambda}$, the assertion is an immediate consequence of the well-known equivalences

$$
Y \subseteq B(L)^{0} \Longleftrightarrow B^{\prime}(Y) \subseteq L^{0} \Longleftrightarrow B(L) \subseteq Y^{0}
$$

for any subset $Y \subseteq M$ and $L \subseteq X$.

It is easy to see that condition (b) implies that the operator $B^{\prime}$ can be applied exactly on $M_{\Lambda}$ w.r.t. $X_{\Lambda}$. In fact, in view of (b), we have

$$
\left\langle B^{\prime} p_{\Lambda}, \psi_{\lambda}\right\rangle=0
$$

for all $p_{\Lambda} \in M_{\Lambda}$ and all $\lambda \in \nabla^{X} \backslash \Lambda^{X}$ and thus

$$
\left\langle B^{\prime} p_{\Lambda}, u\right\rangle=\left\langle B^{\prime} p_{\Lambda}, Q_{\Lambda} u\right\rangle
$$

where $Q_{\Lambda}: X \rightarrow X_{\Lambda}$ denotes the biorthogonal projector onto $X_{\Lambda}$.

\subsection{Full equilibrium}

It is obvious that equilibrated discretizations allow the use of more powerful analytical tools for studying the approximation of saddle point problems. For instance, one may obtain error estimates only for the variable $u$ without using the graph norm, see e.g. [5] and (3.24) above. However, it is in general a non trivial task to realize equilibrated discretizations. Hence, we invest in the development of sufficient criteria when using multiscale bases. Again, it turns out that biorthogonality is a useful tool.

Theorem 5.3. If a multiscale discretization, given by the set of indices $\Lambda=\left(\Lambda^{X}, \Lambda^{M}\right)$ fulfills one of the following equivalent conditions

$$
\begin{gathered}
B\left(X_{\Lambda}\right) \subseteq \tilde{M}_{\Lambda}, \\
B^{\prime}\left(M \ominus M_{\Lambda}\right) \subseteq X^{\prime} \ominus \tilde{X}_{\Lambda},
\end{gathered}
$$

then the discretization is equilibrated.

Proof. Indeed, if (5.10) holds, this means that $\left\langle B v_{\Lambda}, q\right\rangle_{M^{\prime} \times M}=0$ for all $q \in M \ominus M_{\Lambda}$ for any $v_{\Lambda} \in X_{\Lambda}$. This shows that $\left\langle B v_{\Lambda}, q_{\Lambda}\right\rangle_{M^{\prime} \times M}=0$ for all $q_{\Lambda} \in M_{\Lambda}$ already implies $B v_{\Lambda}=0$. The stated equivalence of (5.10) and (5.11) follows by (5.9). 
In turns (5.10) ensures that $B$ can be applied exactly on $X_{\Lambda}$ since

$$
\left\langle B u_{\Lambda}, \vartheta_{\mu}\right\rangle=0
$$

for all $u_{\Lambda} \in X_{\Lambda}$ and all $\mu \in \nabla^{M} \backslash \Lambda^{M}$. We may combine Theorem 5.2 and 5.3, so that we easily obtain the following result.

Corollary 5.4. If the spaces $X_{\Lambda}$ and $M_{\Lambda}$ fulfill $B^{\prime}\left(M_{\Lambda}\right)=\tilde{X}_{\Lambda}$, or equivalently, $B\left(X_{\Lambda}\right)=\tilde{M}_{\Lambda}$, both (LBB) and (FEP) are valid and the operators $B$ and $B^{\prime}$ can be applied exactly w.r.t. $X_{\Lambda}$ and $M_{\Lambda}$.

Let us finally add a comment concerning the relationship of the condition

$$
R B\left(X_{\Lambda_{i}}\right) \subseteq M_{\Lambda_{i}}
$$

in Algorithm 4.3 and (5.10). If $R$ is the Riesz operator, (5.12) is equivalent to

$$
B\left(X_{\Lambda_{i}}\right) \subseteq R\left(M_{\Lambda_{i}}\right)=\tilde{M}_{\Lambda_{i}},
$$

which is exactly condition (5.10). This means that if we use our criteria to enforce (FEP), we automatically fulfill (5.12) and vice versa.

One example. Let us illustrate the above conditions by one simple example. Let us assume that we have two basis functions $\psi_{\lambda_{1}}, \psi_{\lambda_{2}} \in X$, such that $B \psi_{\lambda_{1}}=B \psi_{\lambda_{2}}=c \tilde{\vartheta}_{\mu}$ for some dual basis function $\tilde{\vartheta}_{\mu} \in M^{\prime}$ and some $c \neq 0$. Let us now assume that

$$
\psi_{\lambda_{1}} \in X_{\Lambda}, \quad \psi_{\lambda_{2}} \notin X_{\Lambda}
$$

Using condition (c) in Theorem 5.2 applied to $\psi_{\lambda_{2}}$, it follows that $\vartheta_{\mu} \notin M_{\Lambda}$ for ensuring the LBB condition. On the other hand, using (5.10) applied to $\psi_{\lambda_{1}}$ for checking (FEP), one would obtain $\vartheta_{\mu} \in M_{\Lambda}$ which contradicts the condition (c) in Theorem 5.2. This shows that (5.13) is not possible for a stable and equilibrated discretization. The "inverse" images with respect to $B$ of a certain basis function $\tilde{\vartheta}_{\mu}$ either all have to belong to $X_{\Lambda}$ or none of them.

Remark 5.5. One could try to make use of (FEP) which is a very strong property in order to derive a convergent strategy at least for $u$. Indeed, exploiting (LBB), we obtain for the operator $Q_{\Lambda} \in \mathcal{L}\left(X, X_{\Lambda}\right)$ in Proposition 5.1

$$
b\left(Q_{\Lambda} u-u_{\Lambda}, q_{\Lambda}\right)=b\left(Q_{\Lambda} u, q_{\Lambda}\right)-\left\langle g, q_{\Lambda}\right\rangle_{M^{\prime} \times M}=b\left(u, q_{\Lambda}\right)-\left\langle g, q_{\Lambda}\right\rangle_{M^{\prime} \times M}=0
$$

for all $q_{\Lambda} \in M_{\Lambda}$, where $u, u_{\Lambda}$ are the solutions of the continuous and discrete Problem (2.5), respectively. Hence, (FEP) implies

$$
b\left(Q_{\Lambda} u-u_{\Lambda}, q\right)=0 \quad \text { for all } q \in M .
$$

Since $Q_{\Lambda} u-u_{\Lambda} \in X_{\Lambda}$, we obtain $a\left(Q_{\Lambda} u-u_{\Lambda}, u-u_{\Lambda}\right)=0$, which, in turn, implies

$$
\left\|Q_{\Lambda} u-u\right\|_{A}^{2}=\left\|Q_{\Lambda} u-u_{\Lambda}\right\|_{A}^{2}+\left\|u-u_{\Lambda}\right\|_{A}^{2} .
$$

This latter equation immediately implies

$$
\left\|u-u_{\Lambda}\right\|_{A} \lesssim\left\|u-Q_{\Lambda} u\right\|_{A}
$$

and since $Q_{\Lambda}$ was nothing but the biorthogonal projector on $X_{\Lambda}$, the Riesz basis property ensures that $u_{\Lambda}$ converges to $u$. However, this is also not what we really want to achieve due to two reasons. Firstly, the 
right-hand side of (5.17) contains quantities depending on the unknown solution $u$. This means, the choice of the index sets $\Lambda$ depend directly on $u$, which is not available in numerical calculations. Secondly, (5.17) gives no quantitative estimate which allows to predict the number of iterations an adaptive algorithm has to perform at most to reach a prescribed error tolerance.

Acknowledgements. We would like to thank Franco Brezzi, Albert Cohen, Wolfgang Dahmen and the referees for valuable comments.

\section{REFERENCES}

[1] H.W. Alt, Lineare Funktionalanalysis (in german). Springer-Verlag, Berlin (1985).

[2] K. Arrow, L. Hurwicz and H. Uzawa, Studies in Nonlinear Programming. Stanford University Press, Stanford, CA (1958).

[3] S. Bertoluzza, A posteriori error estimates for the wavelet Galerkin method. Appl. Math. Lett. 8 (1995) 1-6.

[4] S. Bertoluzza and R. Masson, Espaces vitesses-pression d'ondelettes adaptives satisfaisant la condition Inf-Sup. C. R. Acad. Sci. Paris, Sér. Math. 323 (1996).

[5] D. Braess, Finite Elements: Theory, Fast Solvers and Applications in Solid Mechanics. Cambridge University Press, Cambridge (1997).

[6] J.H. Bramble, J.E. Pasciak and A.T. Vassilev, Analysis of the inexact Uzawa algorithm for saddle point problems. SIAM J. Numer. Anal. 34 (1997) 1072-1092.

[7] F. Brezzi and M. Fortin, Mixed and Hybrid Finite Element Methods. Springer-Verlag, New York (1991).

[8] A. Cohen, Wavelet methods in Numerical Analysis, in: Handbook of Numerical Analysis, North Holland, Amsterdam (to appear).

[9] A. Cohen, W. Dahmen and R. DeVore, Adaptive wavelet schemes for elliptic operator equations - Convergence rates, RWTH Aachen, IGPM Preprint 165, 1998. Math. Comput. (to appear).

[10] S. Dahlke, W. Dahmen, R. Hochmuth and R. Schneider, Stable multiscale bases and local error estimation for elliptic problems. Appl. Numer. Math. 23 (1997) 21-48.

[11] S. Dahlke, R. Hochmuth and K. Urban, Adaptive wavelet methods for saddle point problems, Preprint 1126, Istituto di Analisi Numerica del C. N. R. (1999).

[12] S. Dahlke, R. Hochmuth and K. Urban, Convergent Adaptive Wavelet Methods for the Stokes Problem, in: Multigrid Methods VI, E. Dick, K. Riemslagh, J. Vierendeels Eds., Springer-Verlag (2000).

[13] W. Dahmen, Stability of multiscale transformations. J. Fourier Anal. Appl. 2 (1996) 341-361.

[14] W. Dahmen, Wavelet and multiscale methods for operator equations. Acta Numerica 6 (1997) 55-228.

[15] W. Dahmen, Wavelet methods for PDEs - Some recent developments, RWTH Aachen, IGPM Preprint 183 (1999).

[16] W. Dahmen, A. Kunoth and K. Urban, A Wavelet-Galerkin method for the Stokes problem. Computing 56 (1996) $259-302$.

[17] H.C. Elman and G.H. Golub, Inexact and preconditioned Uzawa algorithms for saddle point problems. SIAM J. Numer. Anal. 31 (1994) 1645-1661.

[18] M. Fortin, Old and new Finite Elements for incompressible flows. Int. J. Numer. Meth. Fluids 1 (1981) 347-364.

[19] R. Hochmuth, Stable multiscale discretizations for saddle point problems and preconditioning. Numer. Funct. Anal. and Optimiz. 19 (1998) 789-806.

[20] P.G. Lemarié-Rieusset, Analyses multi-résolutions non orthogonales, Commutation entre Projecteurs et Derivation et Ondelettes Vecteurs à divergence nulle. Rev. Mat. Iberoam. 8 (1992) 221-236.

[21] R. Masson, Wavelet discretizations of the Stokes problem in velocity-pressure variables, Preprint, Univ. P. et M. Curie, Paris (1998).

[22] K. Urban, On divergence-free wavelets. Adv. Comput. Math. 4 (1995) 51-82.

[23] K. Urban, Wavelet bases in $\boldsymbol{H}($ div $)$ and $\boldsymbol{H}(\boldsymbol{c u r l})$, Preprint 1106, Istituto di Analisi Numerica del C. N. R., 1998. Math. Comput. (to appear).

To access this journal online: www.edpsciences.org 